\title{
Enhancement of shearography-based damage identification using best tree wavelet packet analysis
}

\author{
Andrzej Katunin ${ }^{1, *}$, Hernani Lopes ${ }^{2}$, and José Viriato Araújo dos Santos ${ }^{3}$ \\ ${ }^{1}$ Silesian University of Technology, Institute of Fundamentals of Machinery Design, Konarskiego \\ 18A, 44-100 Gliwice, Poland \\ ${ }^{2}$ Instituto Politécnico do Porto, ISEP, DEM, Rua Dr. António Bernardino de Almeida, 431, 4249-015 \\ Porto, Portugal \\ ${ }^{3}$ Universidade de Lisboa, Instituto Superior Técnico, IDMEC, Av. Rovisco Pais, 1049-001 Lisboa, \\ Portugal
}

\begin{abstract}
Shearography found many industrial applications as a nondestructive testing method due to its high spatial resolution and contactless measurements. However, to detect small structural damage, shearography should be enhanced by applying advanced signal processing methods to results of experimental testing. In this paper, the authors present an enhanced method based on the best tree wavelet packet analysis, which allows for extraction of the most informative nodes from the 2D wavelet packet decomposition tree. The proposed method is more effective than typical wavelet transforms due to its ability of adaptive selection of the best basis. The efficiency of the method was verified experimentally on damaged plates. The obtained results clearly show high sensitivity to the introduced small damage, which make the method attractive for industrial applications.
\end{abstract}

\section{Introduction}

Shearography is an interferometric technique that is based on the speckle phenomenon [1]. This technique allows non-contact, full-field and high resolution measurements and measures directly the displacement gradient field in a given direction and is, therefore, almost insensitive to rigid body movements [2]. The applications of shearography range from qualitative non-destructive testing (NDT), strain measurement up to small-scale measurements [3]. Usually one needs a rather large damage to be able to evaluate it directly from the measurements with shearography. Therefore, it is necessary to apply additional signal processing tools in order to quantitatively evaluate small damage.

In [4], it was found out that Gauss differentiation is a suitable technique to compute curvature fields from rotations measured with shearography. The problem of propagation and amplification of noise when computing derivatives was tackled by defining an optimal spatial sampling [5]. Another effective approach for post-processing measured data consists

* Corresponding author: andrzej.katunin@polsl.p1 
in applying various types of wavelet transforms, namely to mode shapes, which was confirmed by numerous studies [6-10]. The wavelet transform, due to its very high sensitivity to disturbances in measured signals, e.g. mode shapes, caused by damage, is an effective processing tool in damage identification. However, in order to improve the effectiveness of damage identification variations of wavelet transforms are of the interest of numerous researchers.

In this study, the authors present a novel approach to enhance damage detectability in plates using shearographic NDT method based on the adapted best tree wavelet packet analysis. This approach allows not only to detect the damage, but also to clearly identify its position. The obtained results indicate that the proposed approach can be an attractive tool for damage identification problems appearing in industry.

\section{Specimen and experimental setup}

The specimen studied in this work is an aluminum plate with a length of $400 \mathrm{~mm}$, a width of $40 \mathrm{~mm}$ and a thickness of $3 \mathrm{~mm}$. In order to locally decrease the bending stiffness, a reduction in the thickness of two areas of the plate was created by a milling machine, as depicted in Figure 1(a). With this process we obtain two slots with depths of $0.41 \mathrm{~mm}$ and $0.30 \mathrm{~mm}$ localized at coordinates $284 \mathrm{~mm}$ and $200 \mathrm{~mm}$, respectively, and with the widths defined in Figure 1(b).

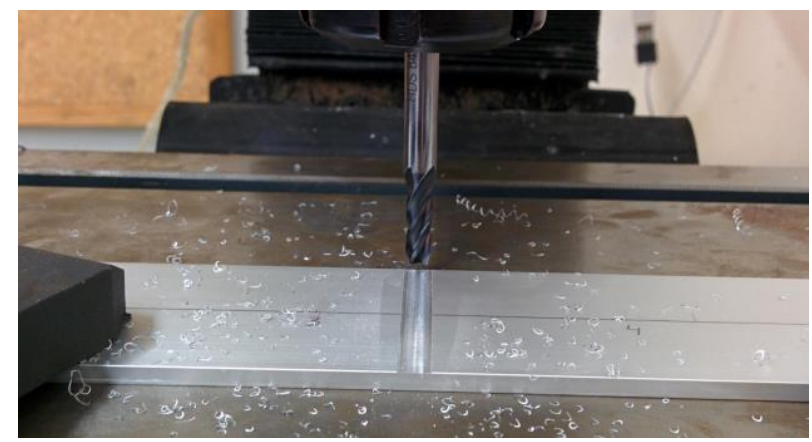

(a) Process of creating the two slots

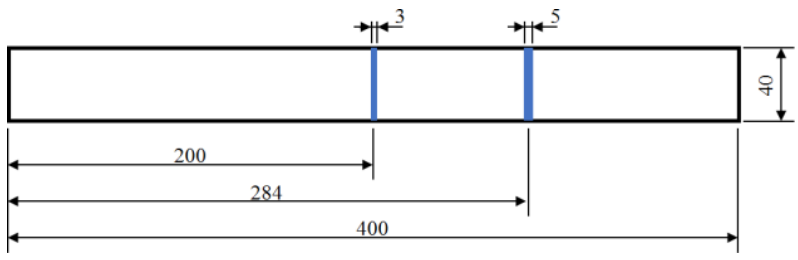

(b) Localization of the two slots

Fig. 1. Characterization of the damage scenario studied.

The modal rotation fields of the first four bending modes were measured using an inhouse built shearographic system that can be seen in Figure 2. The plate is suspended by two rubber bands and is excited acoustically by a loudspeaker. The illumination of the plate surface is made by a continuous-wave laser with a wavelength of $535 \mathrm{~nm}$ and output power of $1.3 \mathrm{~W}$. An acousto-optic modulator is used to pulse the laser and synchronize it with the vibration of the plate. Intensity patterns are recorded by a digital camera with a spatial resolution of 4 million points. A temporal phase shifting is applied in order to extract the phase map from the intensity patterns. A comprehensive description of the experimental setup can be found in [5]. 


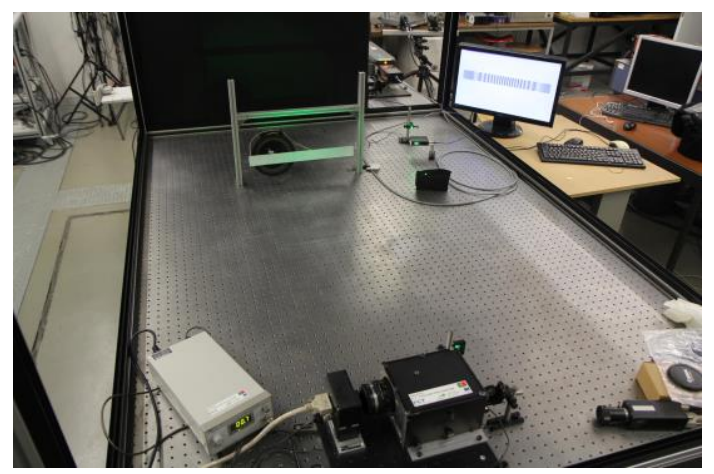

Fig. 2. Experimental setup used for the measurement of the first four modal rotation fields.

The measurement of a modal rotation field involves filtering and unwrapping of a phase map, as shown in Figure 3 for the case of the first modal rotation field relative to the damage scenario depicted in Figure 1(b). It is clear that the plots in Figure 3 do not present any perturbations due to the damage, thus showing that although there is a change in the geometry its signature is not visible. Although not presented here, the other modal rotation fields also do not present a visible signature of the damage.

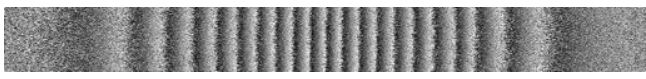

(a) Unfiltered discontinuous phase map

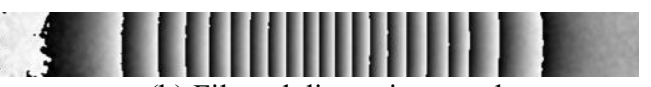

(b) Filtered discontinuous phase map

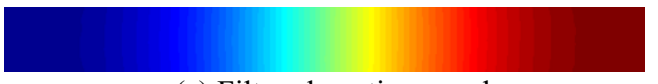

(c) Filtered continuous phase map

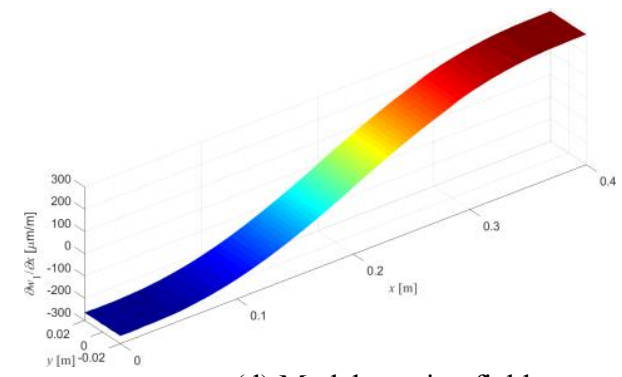

(d) Modal rotation field

Fig. 3. Image processing necessary to obtain a modal rotation field.

\section{Best tree wavelet packet analysis and damage identification}

The 2D wavelet packet transform (WPT), instead of classical 2D multilevel discrete wavelet transform (DWT) results in full decomposition tree, i.e. both approximation and detail sets of coefficients are subjected to further decomposition on subsequent levels, while DWT decomposes approximation sets of coefficients only. Although, WPT is redundant with respect to DWT, it can be more sensitive to disturbances in $2 \mathrm{D}$ signals and, thus, to structural damage, due to its better resolution of the space-frequency representation [11]. This makes WPT a perfect candidate to identify very small non-monotonicities in 2D 
signals, which cannot be even detected by simple observation of the modal rotation fields obtained from shearographic testing.

In order to make further improvement of the effectiveness of the WPT decomposition, the authors of [12] proposed the best decomposition tree algorithm, which is based on the cost function and allows for optimization of the full decomposition tree obtained from WPT. Such an optimization can be performed following several criteria, usually based on entropy or energy measures of sets of coefficients on particular decomposition levels.

In the current study, the entropy type criterion was selected to optimize the WPT decomposition tree and the decomposition level is limited to 3, since decomposition on higher levels resulted in higher disturbance of the final diagnostic 2D signal. The decomposition was performed using B-spline wavelet of order 2 due to its outstanding properties in damage identification problems [11]. The procedure of damage identification was as follows. The modal rotation fields acquired from shearographic testing were subjected to multiresolution WPT for each considered modal rotation field. Considering multiple modal rotation fields is necessary since the magnitude of resulting coefficients is directly related with the magnitude of rotations in a given modal rotation field. In the case when the magnitude of rotations is small, the coefficients are also small, which may mask a damage signature and make it practically undetectable. A unique optimal decomposition tree was obtained for each considered modal rotation field. An example of a full WPT tree and an optimal WPT tree is presented in Figure 4. The optimal decomposition tree approach allows for considering only those sets of coefficients, which contain valuable information from the diagnostic point of view.

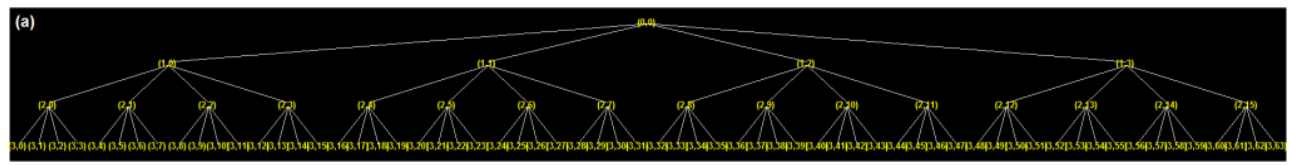

(a) Full tree

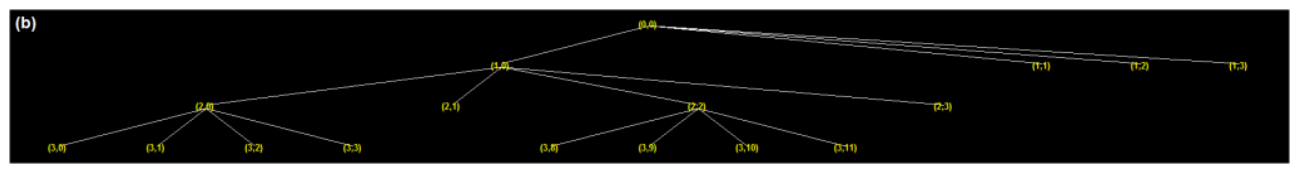

(b) Optimal tree

Fig. 4. Wavelet packet decomposition trees.

Since all the information about possible damage is stored in detail coefficients, only these coefficients were considered in further studies. Each of the selected sets of detail coefficients was subjected to the wavelet packet reconstruction using the same parameters as for the decomposition, and the absolute values of reconstructed coefficients were added to the rest of the stored coefficients for a given modal rotation field. Further, the results for all four modal rotation fields of the tested plate with milled slots were raised to a fractional power in order to emphasize damage signatures, and afterwards merged. The result of performing the above-presented procedure is presented in Figure 5.

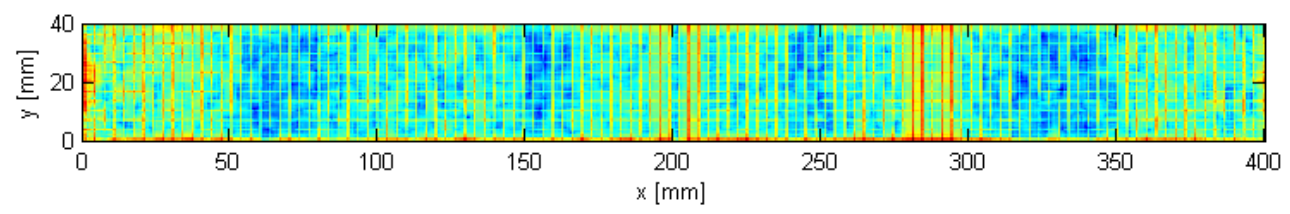

Fig. 5. Result of damage identification procedure obtained using best tree wavelet packet approach. 
From the presented result in Figure 5 one can clearly identify not only the presence of the two introduced slots, but also their boundaries. Therefore, it is clear the great sensitivity of the proposed method to structural damage, thus allowing a significant enhancement of the damage identification ability of shearographic testing.

\section{Conclusions}

Shearographic testing of structures is an NDT method with proven effectiveness, which found an application in various industrial branches, including the aerospace industry. However, in order to detect and identify small damage, additional processing of the resulting $2 \mathrm{D}$ arrays is necessary. One effective approach is the application of waveletrelated transforms due to its high sensitivity to minute sudden changes and minimal disturbances in modal rotation fields caused by damage. The presented study clearly shows that wavelet packet transform with optimization of the decomposition tree allows for effective structural damage identification with simultaneous reduction of processing noise by excluding low-energy sets of coefficients from the decomposition tree. The next studies will be focused on further improvement of this approach in order to create the possibility of detecting even smaller damage.

This publication is supported within the framework of the Rector's grant no. 10/060/RGJ18/0083 in the area of scientific and development research of the Silesian University of Technology, Poland (AK), and LAETA, Project UID/EMS/50022/2013 through IDMEC and INEGI/UP, Portugal (HL, JVAS).

\section{References}

1. W. Steinchen, L. Yang, Digital Shearography: Theory and Application of Digital Speckle Pattern Shearing Interferometry, SPIE Press, 2003

2. Y.Y. Hung, Displacement and strain measurement, in: R.K. Erf (Ed.), Speckle Metrology, Academic Press, New York, 1987, pp. 51-71

3. D. Francis, R. P. Tatam, R. M. Groves, Meas. Sci. Technol. 21, 102001 (2010)

4. H. M. R. Lopes, J. V. A. dos Santos, C. M. M. Soares, R. J. M. Guedes, M. A. P. Vaz, Comput. Struct., 89, 1754-1770 (2011)

5. M. Mininni, S. Gabriele, H. Lopes, J. V. A. dos Santos, Mech. Syst. Signal Pr. 79, 47-64 (2016)

6. M. Rucka, K. Wilde, J. Sound Vib. 297, 536-550 (2006)

7. W. Fan, P. Qiao, Int. J. Solids Struct. 46, 43794395 (2009)

8. A. Katunin, Mech. Syst. Signal Pr. 25, 3153-3167, (2011).

9. M. Cao, L. Cheng, Z. Su, H. Xu, Mech. Syst. Signal Pr. 28, 2012, 638-659.

10. A. Katunin, F. Holewik, Arch. Civil Mech. Eng. 13, 287-296, (2013).

11. A. Katunin, Diagnostics of composite structures using wavelets (The Publishing House of the Institute for Sustainable Technologies - NRI, Radom, 2015)

12. R. R. Coifman, M. V. Wickerhauser, IEEE Trans. Inf. Theor. 38, 713 (1992) 